\title{
Temperature and Wavelength Dependent Emissivity of a Shocked Surface: A First Experiment
}

P. Poulsen and D. E. Hare

This article was submitted to

U.S. Department of Energy

SPIE Thermosense Conference, Orlando, FL; April 1-5, 2002

Lawrence

Livermore

National

Laboratory

January 25, 2002 


\section{DISCLAIMER}

This document was prepared as an account of work sponsored by an agency of the United States Government. Neither the United States Government nor the University of California nor any of their employees, makes any warranty, express or implied, or assumes any legal liability or responsibility for the accuracy, completeness, or usefulness of any information, apparatus, product, or process disclosed, or represents that its use would not infringe privately owned rights. Reference herein to any specific commercial product, process, or service by trade name, trademark, manufacturer, or otherwise, does not necessarily constitute or imply its endorsement, recommendation, or favoring by the United States Government or the University of California. The views and opinions of authors expressed herein do not necessarily state or reflect those of the United States Government or the University of California, and shall not be used for advertising or product endorsement purposes.

This is a preprint of a paper intended for publication in a journal or proceedings. Since changes may be made before publication, this preprint is made available with the understanding that it will not be cited or reproduced without the permission of the author.

This report has been reproduced

directly from the best available copy.

Available to DOE and DOE contractors from the

Office of Scientific and Technical Information

P.O. Box 62, Oak Ridge, TN 37831

Prices available from (423) 576-8401

http: / / apollo.osti.gov/ bridge/

Available to the public from the

National Technical Information Service

U.S. Department of Commerce

5285 Port Royal Rd., Springfield, VA 22161

http:/ / www.ntis.gov/

OR

Lawrence Livermore National Laboratory

Technical Information Department's Digital Library

http: / / www.llnl.gov/tid/Library.html 


\title{
Temperature and wavelength dependent emissivity of a shocked surface: A first experiment
}

\author{
Peter Poulsen* and David E. Hare** \\ Lawrence Livermore National Laboratory
}

\begin{abstract}
We have conducted an experiment in which the temperature and the wavelength dependent emissivity of a shocked surface has been measured. In the past, only the thermal emission from the shocked surface has been measured. The lack of knowledge of the emissivity as a function of wavelength leads to uncertainty in converting the measured emission spectrum into a surface temperature. We have developed a technique by which we are able to calculate both the emissivity of the shocked surface over a range of relevant wavelengths and the temperature of the surface. We use a multi-channel spectrometer in combination with a pulsed light source having a known spectrum of infrared radiation. Two separate techniques using a pulse of reflected radiation are employed and described. Both give the same result: An initially polished molybdenum surface that is shocked and partially released has a temperature of 1040 degrees Kelvin and a wavelength $(\lambda)$ dependent emissivity of $0.16(\lambda=1.2 \mu \mathrm{m}), 0.10(\lambda=1.6 \mu \mathrm{m})$, and $0.20(\lambda=2.3 \mu \mathrm{m})$.
\end{abstract}

Keywords: Radiative thermometry, emissivity measurement

\section{MEASUREMENT OF EMISSIVITY AND TEMPERATURE}

The measurement of the temperature of a material is essential for understanding a wide range of materials processes (e. g. shock dynamics, foundry operations, and wafer manufacture) where knowledge of the state of the material is important. Inferring the temperature from a measurement of radiation is difficult, however. An experiment in which the thermal emission from a shocked material was successfully measured is described by Poulsen, et $\mathrm{al}^{1}$. In this experiment the emitted power from a warm surface is measured over a range of wavelengths using a multi-channel spectrometer. Each channel of the spectrometer passes the radiation from a selected wavelength interval into a detector. The difficulty in the measurement lies in the relation of the emitted power in each channel to the temperature of the surface. The emitted power is determined not only by the temperature of the surface, but also by the emissivity of the surface. The emissivity is not a fixed number for any real surface, but varies both with angle of observation and with wavelength. Thus the temperature cannot be calculated from the emitted power spectrum without detailed knowledge of the emissivity.

It is possible, as shown below, to solve explicitly for the emissivity in each channel wavelength interval as well as for the temperature of the surface. The additional data that make this possible can be either a measurement of the spectrum of light reflected from the surface from a source of known spectral content or a measurement of the reflected power from both the shocked surface and a calibrated surface. Note that it is not necessary to know the absolute values of the flux, either reflected or emitted. This is a great advantage because the exact geometry of the configuration may be rapidly changing or not known at all.

The relations that are used for the calculations assume that we have measured the reflected and emitted power from a number of wavelength bands of the surface. We have previously conducted a demonstration experiment ${ }^{2}$ in which a stationary, steady state surface was used as the source of emitted thermal radiation. We also acquired a fast source (risetime $<0.5 \mu \mathrm{sec}$.) of light that extended into the infrared regime, and used that to acquire the reflectivity data. We know that we can measure the emitted light in $0.1-0.5$ microseconds from previously published experiments ${ }^{1}$. We also know from the same experiments that we can maintain a steady state condition on a shocked surface for approximately one microsecond. We therefore have a tool that is capable of measuring both reflected and emitted light in less than a microsecond and which is therefore applicable to the determination of the temperature and emissivities of a shocked 
surface. This note discusses the results of the gas gun experiment in which we were able to approximate the required conditions and obtain the surface temperature of a shocked surface.

\section{DISCUSSION OF THE METHODS}

We will discuss two related but separate methods for obtaining the emissivities and the surface temperature. As noted above, both methods use a pulse of light incident onto the surface to acquire information about the reflectivity of the surface. In the first method, here named Method I, we only need to know the spectrum of the incident light as measured by the spectrometer. The relations do not require knowledge of the geometry and absolute determination of the emitted and the reflected power as long as the light is measured at the same point where the incident light originates, a condition which can be reasonably well approximated in an experiment. The method does have limitations which originate in the sensitivity of the solution under certain conditions. These conditions, related to the magnitude and variation of the emissivity with wavelength, are discussed below.

In the second method, named Method II, we substitute a surface with a known reflectivity for the surface of interest (the one that will be shocked). We then measure the light reflected from the substituted surface, replace it with the surface of interest, and measure the reflected light from that surface; in this experiment, the measurement takes place after the surface has been has been shocked. The reflectivity and the emissivity as a function of wavelength of the shocked surface is calculated by comparing the two measurements of reflected power. The temperature is then computed by finding the temperature for which the ratio of the measured power to computed power in each wavelength interval is the same. This method is generally applicable with no restrictions on the value of the emissivity other than measurement accuracy. The method does require that the viewing geometry is effectively the same for both the calibration surface and the shocked surface. The shocked surface can move (most do!), but the viewing conditions must be such that the power emitted and reflected is independent of distance. This condition is usually easily satisfied since the surface to be viewed must have a uniform temperature and emissivity within the angle of acceptance of the diagnostic. The disadvantage of this method, of course, is that it may not always be possible to make the substitution of the surface to be measured with the calibration surface.

\subsection{The equations for method I}

This method uses the relative magnitude of the emitted light in each wavelength channel and the incident light spectrum measured in the same channels to compute the channel emissivities and the surface temperature.

The relations for the emitted light are:

$$
S_{i}=\varepsilon_{i} F_{i}(T) K C_{i}
$$

The relations for the reflected light are:

$$
\mathrm{R}_{\mathrm{i}}=\left(1-\varepsilon_{\mathrm{i}}\right) \mathrm{I}_{\mathrm{i}} \mathrm{HC}_{\mathrm{i}}
$$

Note that it has been assumed that the reflectivity is unity minus the emissivity for each wavelength. This is true for each point on the surface as long as the incident light originates at the same point the emitted and reflected light is measured. The definitions of the symbols are as follows:

$\mathrm{H}$ and $\mathrm{K}$ are geometry factors. They can be made to be, and are assumed to be, independent of the wavelength channel.

$\mathrm{i}$ - denotes channel i

$\mathrm{C}_{\mathrm{i}}-$ sensor calibration factor for channel $\mathrm{i}$

$\mathrm{F}_{\mathrm{i}}(\mathrm{T})$ - black body power in channel $\mathrm{i}$

$I_{i}$ - power from flash lamp source in channel $i$

$\varepsilon_{i}$-emissivity of surface in the wavelength interval of channel i

$\mathrm{S}_{\mathrm{i}}-$ emission signal in channel $\mathrm{i}$

$\mathrm{R}_{\mathrm{i}}-$ signal from reflected flash lamp in channel $\mathrm{i}$ 
It is highly desirable to eliminate the geometry factors since we'd like to work with relative values only; the use of relative values, i.e. the spectrum instead of absolute flux, eliminates serious problems with absolute calibration and the instantaneous measurement of geometry. Hence we choose an arbitrary channel " $n$ " and normalize the equations. Typically the chosen channel is the one with good signal resolution for both the emitted and the reflected signal. The equations become:

$$
A_{i}=e_{i} B_{i}(T) \text { and } D_{i}=E_{i}\left(1-e_{i} \varepsilon_{n}\right) /\left(1-\varepsilon_{n}\right)
$$

with $e_{i}=\varepsilon_{i} / \varepsilon_{n}, A_{i}=S_{i} C_{n} / S_{n} C_{I}, B_{i}=F_{i} / F_{n}, D_{i}=R_{i} C_{n} / R_{n} C_{I}$, and $E_{i}=I_{i} / I_{n}$

We know the values of $A_{i}, D_{i}, E_{i}$, and the functional dependence of $B_{i}$ on $T$. This set of equations allows the solution of $e_{i}, \varepsilon_{n}$, and $T$. Every set of three equations yield a temperature and a set of emissivities if the reflectivity relations are used. In the gray body case, the reflectivity relations contribute no information, the relative emissivities are unity, and any two of the equations yield a temperature. The spread of calculated temperatures is a measure of the consistency and accuracy of the data.

The solution to the above equations can under some circumstances be extremely sensitive to variations in the input data and the experimental parameters. It is worth a brief discussion here since the regions of sensitivity in the equations affect the way the experiment should be done. A more detailed discussion of this subject will be published at a later date.

There are two conditions that cause difficulty: When the emissivities are very nearly equal in each channel, and when they are very small. In the first case the solution is clear: The reflectivity measurement is not needed and the calculation of the relative emissions in pairs of channels should each give the same temperature for ideal data input. The second condition is more difficult experimentally. If the emissivities are all small so the reflectivities are near unity, but yet the ratios of the emissivities in pairwise channels differ greatly from unity there is a serious problem that can only be solved by extreme accuracy in the data. The experimenter is advised to estimate the required accuracy prior to designing the experiment.

The emissivity of a surface is a function of both wavelength and the angle to the surface. The multichannel method described above approximates the wavelength dependence in a step-wise fashion; more channels result in greater accuracy as long as decreasing the filter bandwidth does not affect the signal to noise ratio. Otherwise the two factors must be traded off to optimize the diagnostic.

The angular dependence can be dealt with in either of two ways which render the geometry of emission and reflectivity effectively identical. The first way is to have the detector aperture and the source of pulsed light occupy the same point in space. That condition, of course, may not be perfectly satisfied, but closely spaced fiberoptics is good enough. The other way is to employ an integrating, perfectly reflecting enclosure. The latter has the advantage of increasing the effective emissivity and thus the emitted signal. The disadvantage is that it may be difficult to maintain uniform conditions for the shocked surface within the enclosure. For shock experiments, the interaction between the enclosure and the shocked surface may be a source of unwanted light. In other applications the generation of light is not likely to be an issue.

It is noted that the equation set above has no constraints, i. e. there is nothing in the equations that force the emissivities to be positive and less than unity. Only good data will do that, and even then it is important to have an estimate of the errors.

\subsection{The equations for Method II}

A measurement of the reflected power from a surface having a known reflectivity provides a basis for computing the reflectivity of the shocked surface. We use a gold coated surface which has a reflectivity near unity for the range of

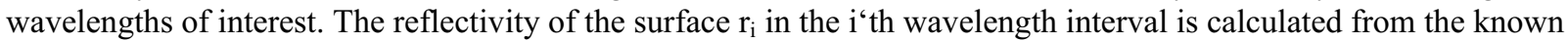
reflectivity $\mathrm{r}_{\mathrm{ci}}$ from the corresponding signals $\mathrm{S}_{\mathrm{i}}$ and $\mathrm{S}_{\mathrm{ci}}$ (subscript $\mathrm{c}$ denotes the calibration surface:

$$
\mathrm{r}_{\mathrm{i}}=\mathrm{S}_{\mathrm{i}} /\left(\mathrm{S}_{\mathrm{ci}} / \mathrm{r}_{\mathrm{ci}}\right)
$$


The calibration factors cancel out in this calculation, so they are not needed. It is important, however, to maintain all conditions other than the surface identical for the two measurements.

Relating the emissivity to the reflectivity, we get

$$
\varepsilon_{\mathrm{i}}=1-\mathrm{r}_{\mathrm{i}}
$$

Knowing the emissivity, we can now calculate the ratio of the measured power to the calculated power. We normalize to the n'th channel to eliminate geometry factors. The computed quantity is $A_{i} / e_{i} B_{i}(T)$ using the above nomenclature. The solution point for the temperature occurs when this quantity has the same value for all channels. Possible variation in the sets of solution points due to measurement errors is discussed below.

\section{THE EXPERIMENT}

A sketch illustrating the experimental arrangement is shown in figure 1. A copper flyer driven by a gas gun to a velocity of $3 \mathrm{~km} / \mathrm{sec}$ impacts a molybdenum plate backed by a lithium fluoride (LiF) window. The LiF window serves two purposes: It increases the pressure at the interface relative to a completely released condition, and it prevents the creation of ejected micro-particles at the surface. The difficulties associated with the $\mathrm{LiF} /$ metal interface are discussed in reference 1.

It is very important in any experiment in which a spectrum is measured to avoid any source of extraneous light. That is accomplished in this experiment by enclosing the $\mathrm{LiF}$ crystal with a non-reflecting surface. The only entry or egress of light is through the optical fibers. Both ends of the fibers must be carefully shielded from extraneous light, of course. As mentioned above, a fully reflecting surface may also be employed with care to integrate over angles and to increase the effective emissivity.
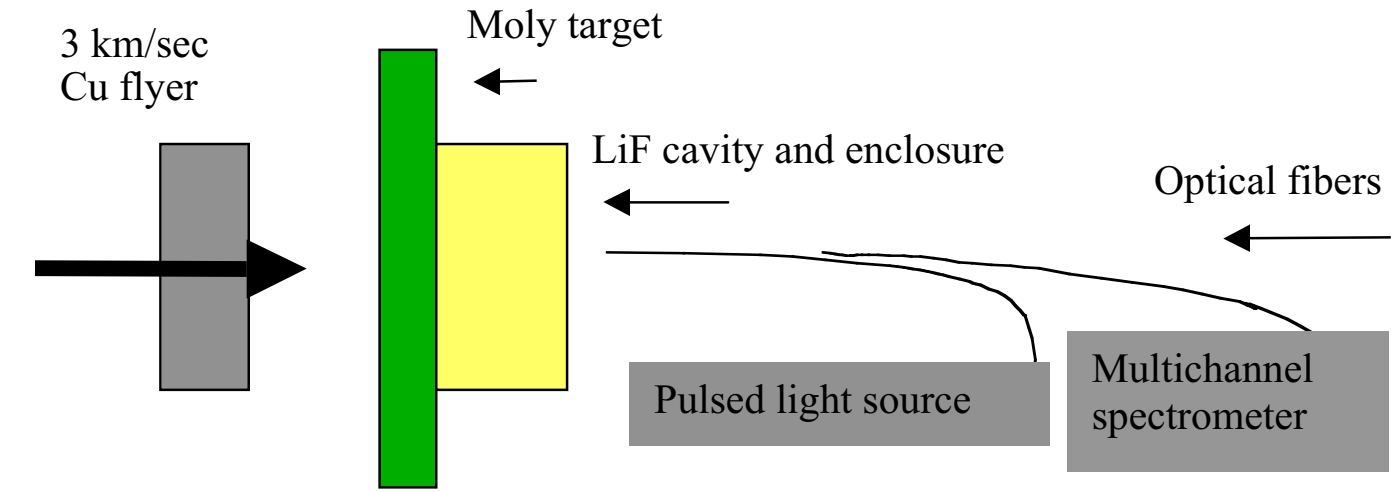

Figure 1: A schematic of the experiment

The idea of the experiment is to shock the moly plate and have the shock reach the interface between the moly and the $\mathrm{LiF}$. The radiation from the shocked surface emits through the $\mathrm{LiF}$ and travels through the fiberoptics to the detectors where the signals are generated. Once a steady state has been reached at the surface (its duration is calculated to be approximately 1.0 microseconds), the source of pulsed light is fired and infrared radiation is reflected off the shocked surface; the sum of the emitted and the reflected radiation is measured by the detectors. In the experiment described below we fired the lamp a little too late because the flyer had a higher speed than expected (we expected $2.6 \mathrm{~km} / \mathrm{sec}$ and measured $3.05 \mathrm{~km} / \mathrm{sec}$ ). A waveform is illustrated in figure 2 below. The intent was to fire the lamp after the emitted signal had been measured, at a point corresponding approximately to -2.0 microseconds and when the peak of the reflected signal was within the steady state region of the signal. We need to be able to subtract the emitted signal from the total signal to obtain the reflected signal. We had reflected signal for the three channels with wavelength less than 3 $\mu \mathrm{m}$. For these data the peak of the reflected light was at $-921 \mathrm{~ns}$, outside of the desired range. Fortunately we found 
from channels at wavelengths in excess of $3 \mu \mathrm{m}$, which have no reflected signal, that the signal was invariant between $-1400 \mathrm{~ns}$ and $-921 \mathrm{~ns}$. Hence we were able to subtract the magnitude of the emitted signal at $-1400 \mathrm{~ns}$ from the sum of the reflected and emitted signal at -921 ns to obtain the reflected signal.. This does introduce the assumption that the temperature and the emissivity in the high wavelength channels is not varying from $-1400 \mathrm{~ns}$ to $-921 \mathrm{~ns}$ in such a manner as to keep the signal constant. Instead we assume that both the emissivity and the temperature are the same at the two times. The self-consistencey of the results that are obtained from the three channels that have a reflected signal supports the assumption. In the future we will time the pulse correctly and no assumptions of this nature will be needed or made.

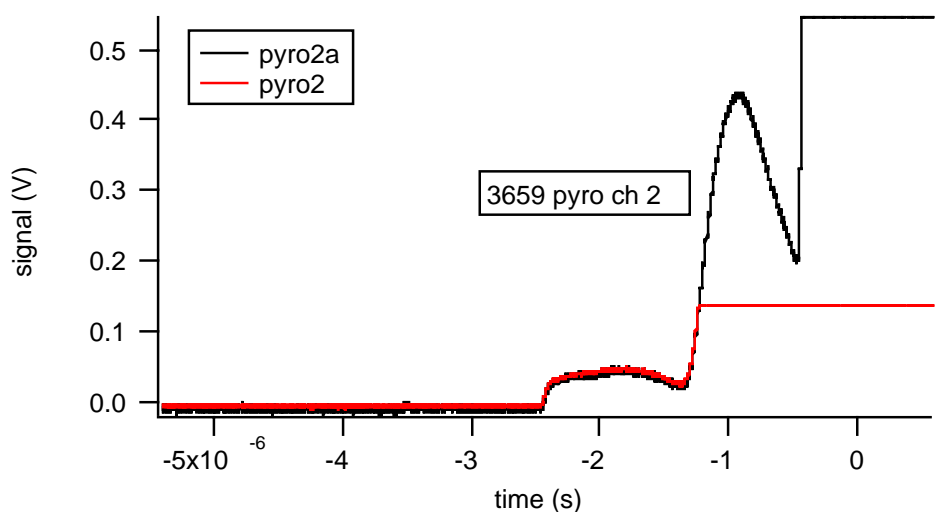

Figure 2: The waveform from the experimental channel 2 detector showing the rise in the signal due to the emitted radiation at approximately -2.4 microseconds. The lamp is fired at approximately -1.3 microseconds, later than desired as discussed in the text.

The target is $6 \mathrm{~mm}$ plate of molybdenum with a polished surface, backed by the LiF crystal. The crystal is completely enclosed in a case which has an aperture for the fiberoptic coupling. Detailed descriptions of the euipment and the data are published in reference 4 . These data were obtained with the LiF enclosure darkened by soot to minimize reflections. This minimized the measurement of radiation originating outside the cone of acceptance of the fiberoptics. It is important to have a surface of uniform temperature within the cone of acceptance of the fiberoptics and to have the geometry of emission the same as the geometry of reflectance. A region of non-uniform temperature is formed at the edge of the impact region due to release processes, and the contribution from this region to the total signal must not be significant if the measurement is to be interpreted by the above analysis methods.

\section{THE DETECTOR AND LIGHT SOURCE}

The detector has 6 channels, each selected for a particular wavelength region. Each channel has an IR detector, optics, a bandpass filter, and passes the light from the surface to the detector via optical fibers. It is similar to the detector described in reference 1 . The configuration of the detector is shown in table 1.

The source of pulsed light is a Hamamatsu xenon high pressure lamp with a sapphire window. It is not perfect for this application because it rises too slowly (about 0.5 microseconds to the peak) and because it has no significant power in the $3-5$ micron wavelength region. We have found it difficult to acquire a source of light in the infrared with the correct properties, and this source allowed us to use three channels and to show that the method works. This is an area that needs improvement for this type of experiment. It is desirable to have a really fast risetime because that allows the use of thinner targets and thus minimizes the effect of edge release on the data. Typical industrial applications do not require measurement times on the order of a microsecond, and adequate sources are available. 
As noted above, the power in the incident light drops off at longer wavelengths. We were unable to use the two large wavelength channels since the reflected power in these channels was insignificant relative to the emitted power.

Table 1. Detector configuration

\begin{tabular}{|l|l|l|l|l|l|}
\hline $\begin{array}{l}\text { Pyro } \\
\text { channel }\end{array}$ & $\begin{array}{l}\text { Analysis } \\
\text { channel }\end{array}$ & filter (um) & lenses & fiber & detector \\
\hline ch1 & NA & NA & NA & NA & NA \\
\hline ch2 & Ch 3 & $\begin{array}{l}1.545- \\
1.742 \text { um }\end{array}$ & silicate glass & low OH FS & InSb \\
\hline ch3 & Ch 4 & $\begin{array}{l}2.004- \\
2.502 \text { um }\end{array}$ & ZnSe & C2 & InSb \\
\hline ch4 & Ch 2 & $\begin{array}{l}1.130- \\
1.348 \text { um }\end{array}$ & $\begin{array}{l}\text { Silicate } \\
\text { glass }\end{array}$ & low OH FS & InSb \\
\hline ch5 & Ch 6 & $\begin{array}{l}2.985- \\
4.979 \text { um }\end{array}$ & ZnSe & C2 & InSb \\
\hline ch6 & Ch 5 & $\begin{array}{l}2.503- \\
3.490 \text { um }\end{array}$ & ZnSe & C2 & HgCdTe \\
\hline $\begin{array}{l}\text { lamp fiber } \\
\text { (four) }\end{array}$ & & & & C2 & \\
\hline
\end{tabular}

Table 2. Times and signal levels used in the analysis

$\begin{array}{lll}\begin{array}{l}\text { Analysis } \\ \text { ch } 2\end{array} & \text { time } & \text { signal } \\ & \begin{array}{ll} \\ \text { ch } 3\end{array} & -1.4 \mathrm{us} \\ & -921 . \mathrm{ns} * & 892 . \mathrm{mV} \\ & -1.4 \mathrm{us} & \\ \text { ch } 4 & -921 . \mathrm{ns} & 32.6 \\ & -1.4 \mathrm{us} & 444 . \mathrm{mV} \\ & -921 . \mathrm{ns} & 118 . \mathrm{mV}\end{array}$

*Note: $-921 \mathrm{~ns}$ is peak of signal in channel 2

The calibration factors $\mathrm{C}_{\mathrm{i}} / \mathrm{C}_{4}$ for analysis channels 2,3 , and 4 were $3.30,2.33$, and 1.00 . The incident signals were 1030 $\mathrm{mV}, 458 \mathrm{mV}$, and $143 \mathrm{mV}$ for analysis channels 2,3 , and 4 .

\section{THE DATA ANALYSIS}

The data was analyzed using the analysis and the calibration detailed above. We will first show the results using Method I. In this method we solve for the emissivity in channel 4 and the surface temperature. In this particular example we only use three channels (analysis channels 2, 3, and 4) since the light reflected from the surface in the other channels is insignificant in relation to the emitted light and cannot be determined with sufficient accuracy. Due to the normalization against channel 4 , the three channels only produces two curves of the emissivity in channel 4 versus the surface temperature, so there will be only one point of intersection. The curves are shown in figure 3 . The point of intersection is at a temperature of 1140 degrees Kelvin and a channel 4 emissivity of 0.20 . 
Using the temperature of 1140 degrees Kelvin and the channel 4 emissivity of 0.20 we can now use the equations shown previously to solve for the emissivities in channels 2 and 3 . They are 0.16 and 0.10 , respectively. The complete solution is shown in table 3. It is noted that the emissivity is a strong and irregular function of wavelength. With such a large variation in emissivity the assumption of a gray body emission (emissivities independent of wavelength) is not warranted and would produce inaccurate results. The data will be analyzed below using a gray body assumption to contrast the results.

Table 3. Temperature and emissivities

\begin{tabular}{|l|c|}
\hline Temperature $\mathrm{K}$ & 1140 \\
\hline Emissivity ch. 2 & 0.16 \\
\hline Emissivity ch. 3 & 0.10 \\
\hline Emissivity ch. 4 & 0.20 \\
\hline
\end{tabular}

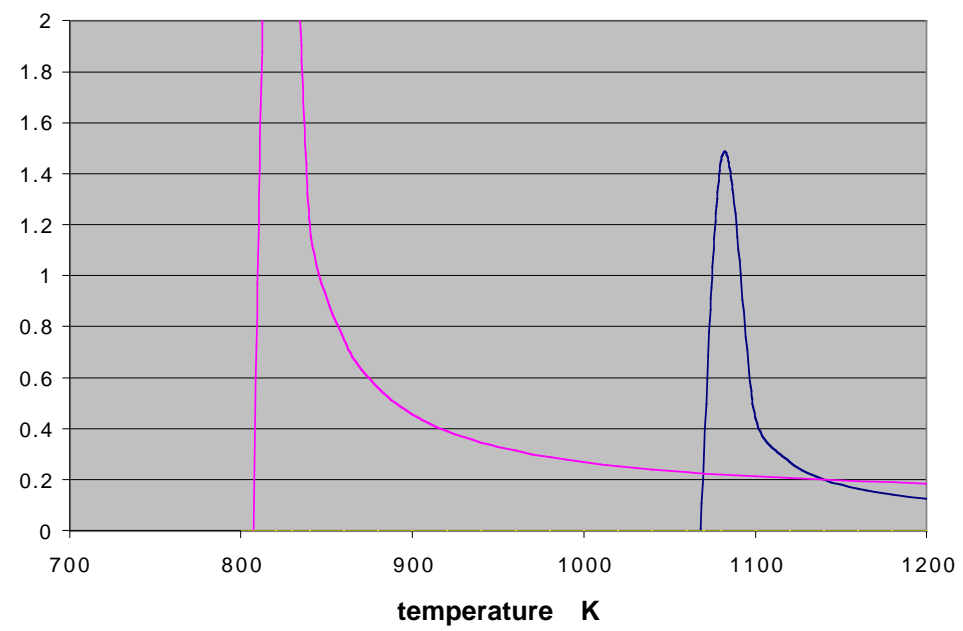

Figure 3. Curves of the emissivity in channel 4 versus the surface temperature in degrees Kelvin for shot 3659 . The solution point is at a temperature of 1140 degrees Kelvin and the emissivity in channel 4 is 0.20 . The vertical parts of the curves are a plotting artifact connecting negative and positive infinities.

We now turn to Method II. For this method to work we first substitute a highly reflecting surface for the moly surface. It is assumed here that it is perfectly reflecting; its absolute reflectivity can be incorporated in the calculations if it is significantly different from unity. From a direct measurement of the absolute values of the reflected signal from the substituted and the shocked surface in each channel we calculate the ratio of the two values in each channels and obtain the reflectivity in each channel. Subtracting this value from unity yields the emissivities in each channel. They are the same as in table 3 to two significant figures.

To determine the temperature in method II we calculate the measured power in each channel divided by the computed power as a function of temperature. We avoid the use of geometrical factors by dividing this ratio by the corresponding ratio for one of the channels; we use channel 4 again. The solution point should ideally be found at the point where all curves cross unity. In real life there will be some variation and a minimization procedure can be used, similar to that used for the gray body calculation. The plot for Method II for these data is shown in figure 4 . The curves cross unity at a temperature of 1140 degrees Kelvin, so the result obtained by this method is the same as for Method I. Measurement errors can prevent the curves from meeting at the same point, thus lending uncertainty to the accuracy of the measured temperature. In this case the error can be estimated using the square root of the sum of the square of the pairwise 
difference in the plotted quantity as a function of temperature. This error can then be related to the uncertainty in the temperature measurement.

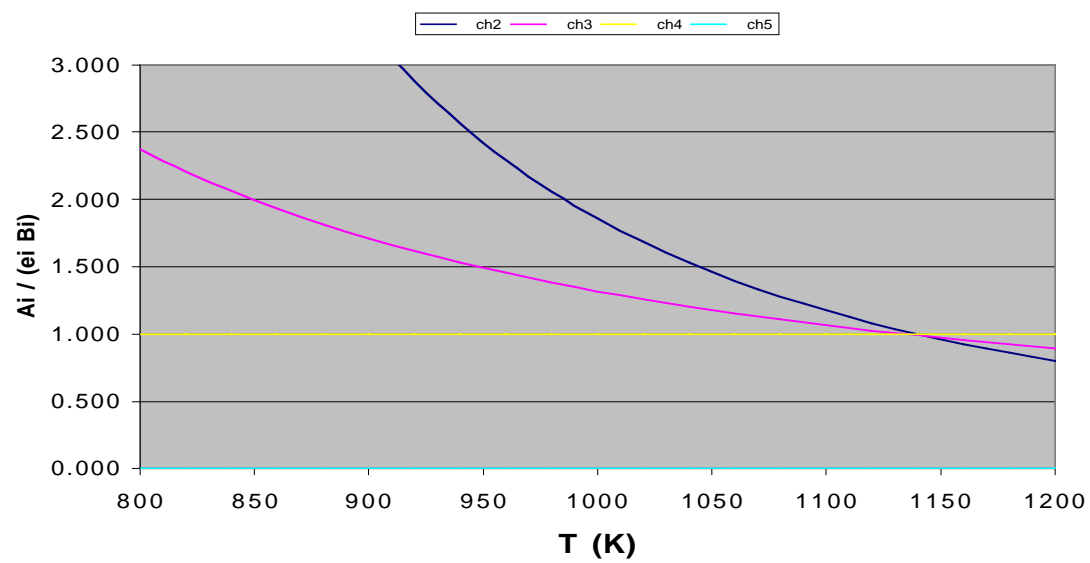

Figure 4. The normalized ratios of measured to calculated emitted power as a function of temperature

An often used method of analysis, the Gray body method, assumes that the emissivity is invariant with wavelength. Only the emission data is used in this analysis. We make a plot of the normalized ratios of measured to calculated emitted power as a function of temperature in a manner similar to that used for figure 4. If the plot shows that the curves meet at the same point, the assumption of equal emissivities is validated. If not, there is no way to know what the temperature really is. A solution point occurs at approximately $850 \mathrm{~K}, 1100 \mathrm{~K}$, and $1450 \mathrm{~K}$. Therefore, the solution points do not validate the assumption of equal emissivities, and the temperature of the surface cannot be accurately determined from these data using a gray body assumption. Using more channels does not aid in finding a solution. We conclude that for these data the Gray body analysis is not valid.

\section{FUTURE WORK}

A number of improvements can be made to the experiment. The risetime of the incident pulse of light can be made much less than the present $0.5 \mu \mathrm{s}$; times on the order of $0.1 \mu \mathrm{s}$ should be achievable. The source used for this experiment can be made faster and we have also acquired an alternative source. In addition, it is highly desirable to extend the range of the incident spectrum to greater wavelengths, at least up to $5 \mu \mathrm{m}$. The LiF crystal does not pass light very well beyond 5 $\mu \mathrm{m}$, so another crystal is needed to extend the range. Under some circumstances, the experiment can be done on a shocked surface without a crystal. The reasons for using a crystal for shocked surface experiments is to suppress ejecta and to maintain a high pressure at the shocked surface.

Improvements in risetime and accuracy of timing will diminish the length of time required to have the surface in a steady state. Decreasing the length of time below the present one microsecond will enable the use of thinner targets and decrease the effect of the release at the edge of the impact zone, thus improving the uniformity of the region viewed by the detector.

This experiment used a near non-reflecting enclosure and depended on its success on a geometrical relationship between the emitter, the light source, and the viewing optics. We are also investigating the use of a perfectly reflecting enclosure; the purpose of this enclosure is to integrate the emission over angles and to increase the effective emissivity of the surface. The latter feature is useful under conditions where the light emitted is otherwise marginal for measurement. 


\section{CONCLUSIONS}

We have shown that the channel emissivities and the temperature of a shocked surface can be determined accurately and self consistently by measuring both reflected and emitted light in each band of wavelengths of a multichannel spectrometer. At least three bands are required. Two independent methods have been used. One method uses only the knowledge of the spectrum of the incident light as well as the emitted and reflected light. The other method requires a calibration of the reflected light from a surface having a known reflectivity (such as gold) in an identical geometry. The first method can be used when the emissivities vary sufficiently with wavelength. If the emissivities do not vary, the Gray body assumptions can be used for the analysis and will yield the correct results. The second method can be used under any conditions, assuming, of course, that the measurement of the known surface can be accomplished.

\section{ACKNOWLEDGMENTS}

We acknowledge the support of Dennis Baum, the advice and assistance of Neil Holmes, Peter Fiske (formerly of LLNL) and David Holtkamp (LANL) in previous experiments. Michael Hiltl (formerly of LLNL) procured and assembled the components of the detector. The experimental work was done by target builder Timothy M. Uphaus, gunners Keith Stickle and Leon C. Raper, and electronics technician Steven J. Caldwell. This work was performed under the auspices of the U.S. Department of Energy by the University of California, Lawrence Livermore National Laboratory under contract No. W-7405-Eng-48.

\section{REFERENCES}

1. P. Poulsen, D. Baum, P. Fiske, and D. Holtkamp, "Temperature Measurement of Shocked Surfaces ". UCRLJC-139909

2. P. Poulsen, D. Hare, and M. Hiltl, "Sub-Microsecond Temperature and Emissivity Measurements", internal memo, LLNL. April 11, 2001. To be published as Lawrence Livermore national Laboratory report (UCRL).

3. J. U. Cazamias, D. Hare, and P. Poulsen, "Progress in Infrared Pyrometry Measurements of Shocked Solids", UCRL-JC-146049

4. P. Poulsen and D. E. Hare, "Temperature and Emissivity of a Shocked Surface: A First Experiment", to be published as Lawrence Livermore national Laboratory report (UCRL).

*poulsen1@1lnl.gov; phone 925-422-6692; Lawrence livermore National laboratory, P.O. box 808, Livermore, CA, USA 94550; **hare2@1lnl.gov, 925-423-6828; Lawrence livermore National laboratory, P.O. box 808, Livermore, CA, USA 94550 